\title{
Regulation of myotube formation by the actin- binding factor drebrin
}

\author{
Annalisa Mancini ${ }^{1}$, Dario Sirabella', Weijia Zhang ${ }^{2}$, Hiroyuki Yamazaki ${ }^{3}$, Tomoaki Shirao ${ }^{3}$ and Robert S Krauss ${ }^{1 *}$
}

\begin{abstract}
Background: Myogenic differentiation involves cell-cycle arrest, activation of the muscle-specific transcriptome, and elongation, alignment and fusion of myoblasts into multinucleated myotubes. This process is controlled by promyogenic transcription factors and regulated by signaling pathways in response to extracellular cues. The p38 mitogen-activated protein kinase (p38 MAPK) pathway promotes the activity of several such transcription factors, including MyoD and MEF2, thereby controlling the muscle-specific transcription program. However, few p38regulated genes that play a role in the regulation of myogenesis have been identified.

Methods: RNA interference (RNAi), chemical inhibition and immunofluorescence approaches were used to assess the role of drebrin in differentiation of primary mouse myoblasts and $\mathrm{C2} 12$ cells.

Results: In a search for p38-regulated genes that promote myogenic differentiation, we identified Dbn1, which encodes the actin-binding protein drebrin. Drebrin is an F-actin side-binding protein that remodels actin to facilitate the change of filopodia into dendritic spines during synaptogenesis in developing neurons. Dbn1 mRNA and protein are induced during differentiation of primary mouse and C2C12 myoblasts, and induction is substantially reduced by the p38 MAPK inhibitor SB203580. Primary myoblasts and C2C12 cells depleted of drebrin by RNAi display reduced levels of myogenin and myosin heavy chain and form multinucleated myotubes very inefficiently. Treatment of myoblasts with BTP2, a small-molecule inhibitor of drebrin, produces a phenotype similar to that produced by knockdown of drebrin, and the inhibitory effects of BTP2 are rescued by expression of a mutant form of drebrin that is unable to bind BTP2. Drebrin in myoblasts is enriched in cellular projections and cell cortices and at regions of cell-cell contact, all sites where F-actin, too, was concentrated.
\end{abstract}

Conclusions: Our findings reveal that Dbn1 expression is a target of p38 MAPK signaling during myogenesis and that drebrin promotes myoblast differentiation.

Keywords: myoblast, cell differentiation, drebrin, myotube, actin

\section{Background}

Myoblast differentiation is a multistep process that involves withdrawal from the cell cycle, acquisition of a cell type-specific transcriptional program and morphological changes that include elongation, alignment and fusion of myoblasts to form myofibers [1-4]. Whereas transcriptional regulation is at the core of myogenesis, the formation and growth of myofibers is also controlled by a variety of signaling ligands and their receptors, including insulin-like growth factor 1 , fibroblast growth

\footnotetext{
* Correspondence: Robert.Krauss@mssm.edu

'Department of Developmental and Regenerative Biology, Mount Sinai School of Medicine, One Gustave L. Levy Place, New York, NY 10029, USA Full list of author information is available at the end of the article
}

factors (FGFs), Wnts, transforming growth factor $\beta$ superfamily members and others [1-3,5]. Furthermore, the activity of MyoD and other promyogenic transcription factors is tightly controlled at the posttranslational level by signal transduction pathways, including phosphatidylinositol 3-kinase/Akt, integrin/focal adhesion kinase (FAK) and calcium/calcineurin [4,6-9]. Among the signaling pathways that promote myogenesis, the $\mathrm{p} 38 \alpha / \beta$ MAPK $(\mathrm{p} 38 \alpha / \beta$ mitogen-activated protein kinase) pathway plays a prominent role. There is a persistent rise in $\mathrm{p} 38 \alpha / \beta$ (hereafter simply "p38") activity during myoblast differentiation, and inhibition of $\mathrm{p} 38$ expression or activity blocks induction of select musclespecific genes and myogenic differentiation [8,10-13].

C Biomed Central

(c) 2011 Mancini et al; licensee BioMed Central Ltd. This is an Open Access article distributed under the terms of the Creative Commons Attribution License (http://creativecommons.org/licenses/by/2.0), which permits unrestricted use, distribution, and reproduction in any medium, provided the original work is properly cited. 
p38 phosphorylates substrates that drive muscle-specific gene expression at several levels, including MyoD dimerization with E proteins, Mef2 transcriptional activity, chromatin remodeling at muscle-specific genes and stability of myogenic mRNAs [11,13-18]. Despite progress in understanding these proximal targets of $\mathrm{p} 38$ 's promyogenic actions, few p38-regulated genes that play a role in the regulation of myogenesis have been identified.

In addition to the acquisition of a muscle-specific transcriptional program, the changes in myoblast morphology that occur during differentiation indicate that dramatic alterations in the F-actin cytoskeleton are required for the formation of myofibers. Consistent with this notion, lamellipodia and filopodia, cellular structures that require actin remodeling, form dynamically during myoblast differentiation in vitro [19-21]. Furthermore, disruption of the Factin cytoskeleton by chemical or other means inhibits various aspects of myoblast differentiation, including myoblast fusion [21-24]. At least some aspects of the cytoskeletal rearrangement and morphological changes that occur during differentiation are likely to be mediated by transcriptional induction of regulators of these processes, as expression of MyoD in fibroblasts induces not only expression of muscle-specific genes but also elongation and fusion into multinucleated myotubes [4].

Drebrin is a ubiquitously expressed F-actin side-binding protein that is highly abundant in the brain $[25,26]$. Drebrin contains an actin-depolymerizing factor II/cofilin-like domain, an actin-binding domain and two Homer-binding domains [27], and it remodels actin to facilitate the maturation of filopodia into dendritic spines during synaptogenesis in developing neurons (for reviews, see $[27,28])$. It is localized in lamellipodia and filopodia, at sites of cell-cell contact and in adhesion plaques [27-32]. Furthermore, drebrin associates with several proteins that promote myoblast differentiation and/or fusion, including the microtubule plus-tip binding protein EB3 [33,34]; the scaffold protein Homer $[35,36]$ and, via Homer, the small GTPase Cdc42 $[35,37,38]$; and the chemokine receptor CXCR4 $[39,40]$. We report herein that drebrin expression is induced during differentiation of primary and $\mathrm{C} 2 \mathrm{C} 12$ myoblasts in a p38 MAPK-dependent manner. Furthermore, depletion of drebrin by RNA interference (RNAi) or inhibition of its function with a small-molecule antagonist diminished expression of muscle-specific genes and myotube formation. Drebrin is therefore an actin-regulating factor induced during myogenic differentiation that promotes the differentiation process.

\section{Methods}

\section{Cell culture}

Primary myoblasts were isolated from wild-type mice at postnatal day 13 by the method of Rando and Blau [41] and maintained as previously described [42]. Differentiation was induced by switching cultures from Ham's F-10 medium with $20 \%$ fetal bovine serum (FBS) $/ 2.5 \mathrm{ng} / \mathrm{ml}$ basic FGF (R\&D Systems, Minneapolis, MN, USA)/4\% penicillin-streptomycin (growth medium $(\mathrm{GM})$ ) to DMEM with $5 \%$ horse serum (differentiation medium (DM)). C2C12 myoblasts were maintained in DMEM containing 15\% FBS supplemented with $1 \%$ penicillin/ streptomycin and L-glutamine (GM). Differentiation was induced by transferring to DMEM supplemented with $2 \%$ horse serum (DM). Where indicated, $5 \mu \mathrm{M}$ SB203580 (Sigma-Aldrich, St Louis, MO, USA) or $5 \mu \mathrm{M}$ BTP2 (Calbiochem, La Jolla, CA, USA) were added to cultures and replenished every 12 hours.

\section{Expression of drebrin}

Plasmids encoding murine GFP-tagged drebrin E were generated by subcloning cDNA encoding either wildtype murine Dbn1 E or the K270M, K271M mutant into the pEGFP-C1 vector (Clontech Laboratories, Inc, Mountain View, CA, USA). Plasmids were transfected into $\mathrm{C} 2 \mathrm{C} 12$ cells using Lipofectamine 2000 reagent (Invitrogen/Life Technologies, Carlsbad, CA, USA) according to the manufacturer's instructions.

\section{RNAi}

For small hairpin RNA studies, four sequences against murine $D b n 1$ were initially chosen. The oligonucleotides were cloned into the pSUPER.puro vector (Oligoengine, Seattle, WA, USA) and transfected into C2C12 cells with Lipofectamine 2000 reagent. Puromycin-resistant cells were selected, pooled and examined by Western blot analysis for drebrin expression. The most effective sequences were chosen for further studies and corresponded to nucleotides 5' GCCACTTCGAGAACCAGAAAG 3' (siDbn1-1) and 5' AGGAAGAGCCATGT GCAAAGGT 3' (siDbn1-3) (NM_019813.3).

Twenty-four hours after selection, cultures were seeded onto growth factor-reduced Matrigel (R\&D Systems)-coated dishes at a density of 150,000 cells $/ \mathrm{ml}$. Twenty-four hours later, cultures were switched to DM. At various time points later, cultures were fixed with $2 \%$ paraformaldehyde (PFA) and immunostained for myosin heavy chain $(\mathrm{MHC})$ and drebrin expression. Sister cultures were lysed, and $30 \mu \mathrm{g}$ of cleared cell extracts were examined by Western blot analysis as previously described [43]. Drebrin expression was also knocked down by transfection of $\mathrm{C} 2 \mathrm{C} 12$ cells or primary myoblasts with 200 nM drebrin-specific RNAi (4390771; Ambion/Life Technologies) or with $200 \mathrm{nM}$ control nonsilencing RNAi Red (465318; Invitrogen/Life Technologies). RNAs were transfected with StemPro LipoMax reagent (Invitrogen/Life Technologies) according to the manufacturer's instructions. Thirty-six hours after 
transfection, cells were either harvested to assess drebrin expression by Western blot analysis or analyzed for differentiation potential tested by transfer to DM.

\section{Protein analysis}

Western blot analyses were performed as described in Kang et al. [43]. Briefly, cells were lysed in extraction buffer (50 mM Tris. $\mathrm{HCl}, \mathrm{pH}$ 7.4, $50 \mathrm{mM} \mathrm{NaF,} 5 \mathrm{mM}$ sodium pyrophosphate, $1 \mathrm{mM}$ sodium orthovanadate, 1 $\mathrm{mM}$ ethylenediaminetetraacetic acid, 1\% Triton X-100) supplemented with protease and phosphatase inhibitor cocktail (Sigma-Aldrich). Total proteins were resolved on SDS-polyacrylamide gels, transferred onto Immobilon polyvinylidene fluoride membranes (Millipore, Billerica, MA, USA) and probed with specific antibodies. Primary antibodies used were anti-drebrin (Abcam, Cambridge, MA, USA), anti-tubulin (Santa Cruz Biotechnology, Santa Cruz, CA, USA), anti-myogenin (F5D; Santa Cruz Biotechnology) and anti-MHC (MF20; Developmental Studies Hybridoma Bank, Department of Biology, University of Iowa, Iowa City, IA, USA). Membranes were reprobed with the appropriate horseradish peroxidase-conjugated secondary antibody (The Jackson Laboratory, Bar Harbor, ME, USA), and specific bands were visualized with an enhanced chemiluminescence detection system (Roche Applied Science, Indianapolis, IN, USA)

\section{RNA analysis}

RNA was isolated using the RNeasy Mini Kit (QIAGEN, Valencia, CA, USA). One microgram of total RNA was reverse-transcribed using the First-Strand cDNA Synthesis Kit (Invitrogen/Life Technologies). One-tenth of the cDNA was applied for real-time PCR using QuantiFast SYBR Green RT-PCR Kit (QIAGEN) and analyzed on a Bio-Rad Q5 cycler (Bio-Rad Laboratories, Hercules, CA, USA).

Dbn1, Myh3, Myog and Gapdh mRNA expression were quantified with the following primers. $D b n 1$ forward: 5' AGGCCAAGAAGGAGGAAGAG 3'; $D b n 1$ reverse: 5' TTCCTCCTGTGCTCCTCAAT 3'; Myh3 forward: 5' CAGAAATGGAGACACGGATCAGA 3'; Myh3 reverse: 5' AGAGGTGAAGTCACGGGTCTT TGCC 3'; Myog forward: 5'GGGCCCCTGGAA GAAAAG 3'; Myog reverse: 5'AGGAGGCGCTGTGGGAGT 3'; Gapdh forward: 5' TGCACCACCAACTGC TTA 3'; Gapdh reverse: 5' GATGCAGGGATGA TGTTC 3'.

\section{Immunostaining and microscopy}

Cells were grown on either Matrigel- or collagen-coated dishes as indicated, fixed with 2\% PFA for 10 minutes, permeabilized with $0.1 \%$ Triton $\mathrm{X}-100$ for 5 minutes, blocked with PBS containing 5\% goat serum for 1 hour and incubated overnight with the following primary antibodies: anti-MHC (clone MF-20; Developmental Studies Hybridoma Bank), anti-drebrin (rabbit; Abnova, Walnut, CA, USA) and Alexa Fluor 568 phalloidin (Invitrogen/Life Technologies). After being washed three times with PBS, cells were incubated with the appropriate secondary antibody conjugated with Alexa Fluor 488 or 568 (Invitrogen/Life Technologies) for 1 hour, counterstained with 4',6-diamidino-2-phenylindole (DAPI) and then visualized using a Nikon Eclipse TS100 inverted microscope (Nikon Instruments, Melville, NY, USA). Images were acquired using a ProgRes digital microscope camera system and software (Jenoptik AG, Jena, Germany).

\section{Cell proliferation and cell death assays}

Cell proliferation was assessed by bromodeoxyuridine (BrdU) incorporation as described by Kang et al. [44]. Briefly, dividing cells were incubated in $20 \mathrm{mM}$ BrdU for 2 hours. The medium was aspirated, and cells were immediately fixed for 30 minutes at $-20^{\circ} \mathrm{C}$ with ice-cold $70 \%$ ethanol $/ 30 \%$ glycine at $\mathrm{pH} 2.0$. After being washed with PBS, cells were incubated with mouse anti-BrdU (Chemicon International/Millipore, Temecula, CA, USA) and anti-mouse Alexa Fluor 568 (Invitrogen/Life Technologies). Cells were counterstained with DAPI and visualized by the standard immunofluorescence protocol. Apoptosis was analyzed with the In Situ Cell Death Detection Kit (Roche Applied Science) according to the manufacturer's instructions.

\section{Results}

Dbn 1 is induced during myogenic differentiation

In an effort to identify genes regulated by p38 signaling during myoblast differentiation, microarray analyses were performed on $\mathrm{C} 2 \mathrm{C} 12$ cells cultured at low density in GM or DM at various time points, plus or minus the p38 inhibitor SB203580. Details of the screen will be published elsewhere. Herein we describe studies of one such gene identified in this screen, $D b n 1$, which encodes drebrin. Quantitative RT-PCR (qRT-PCR) analysis revealed that $D b n 1$ is expressed at very low levels in proliferating primary myoblasts in GM, is expressed at higher levels at the time of shift to DM (day 0, when cells have reached near-confluence) and increases in abundance over the next 2 days in DM (Figure 1A). Analysis of Myog and Myh3 expression served as a positive control for differentiation. $D b n 1$ expression during myoblast differentiation was biphasic, as mRNA levels decreased again after 3 days in DM. Parallel cultures treated with SB203580 displayed strongly dampened induction of Dbn1, Myog and Myh3 expression over this time course (Figure 1A). There are two alternatively spliced isoforms of 

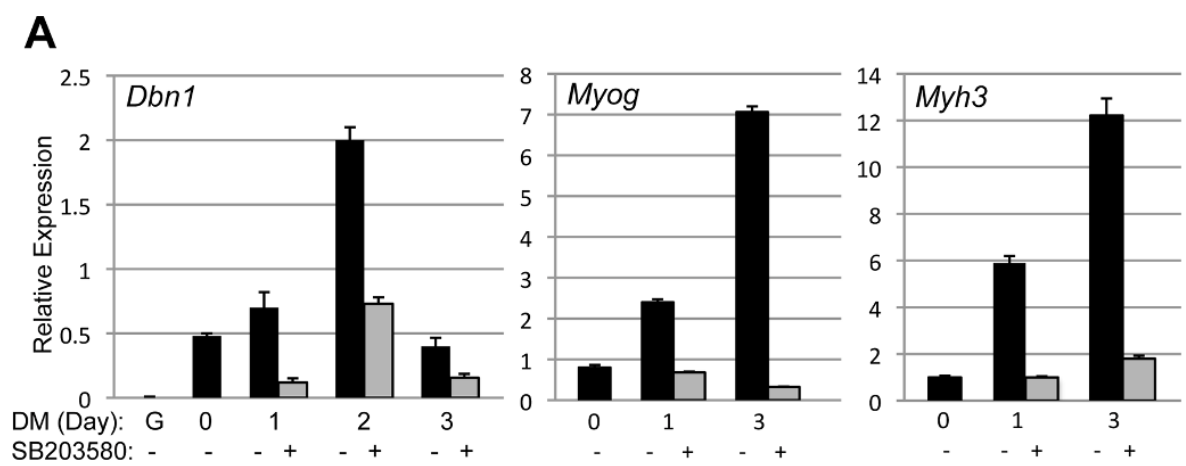

B

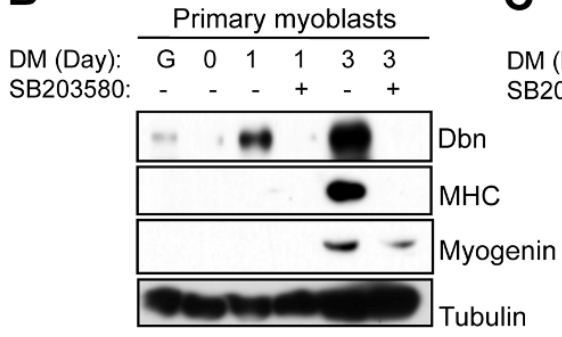

C

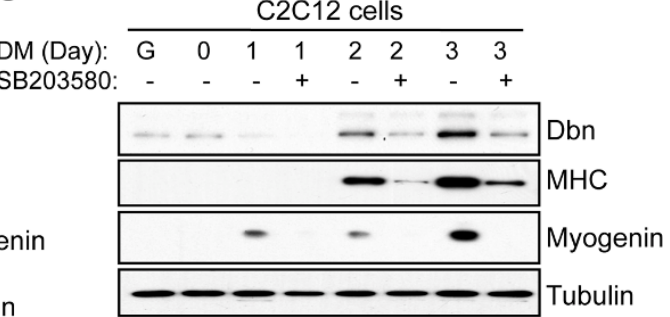

Figure 1 Dbn 1 is upregulated during myoblast differentiation in an SB203580-sensitive manner. (A) Primary mouse myoblasts proliferating in growth medium (G) or transferred to differentiation medium for the indicated times in the presence $(+)$ or absence $(-)$ of $5 \mu M$ SB203580 were analyzed by quantitative RT-PCR for expression Dbn1, Myog (encoding myogenin) and Myh3 (encoding myosin heavy chain 3; MHC3). (B) Cultures parallel to those in part (A) were examined by Western blot analysis for drebrin, myogenin and MHC production. Tubulin was used as a loading control. (C) C2C12 myoblasts were analyzed similarly to the primary myoblasts shown in part (B).

drebrin: A, which contains a brain-specific exon, and E, which lacks this exon and is expressed broadly $[27,28]$. qRT-PCR with pan-drebrin or drebrin A-specific primers revealed, as expected, that drebrin $E$ is the isoform expressed in myoblasts (data not shown). Drebrin protein levels in differentiating cultures of primary myoblasts and $\mathrm{C} 2 \mathrm{C} 12$ cells were regulated similarly to $D b n 1 \mathrm{mRNA}$ in that they were induced during differentiation in an SB203580-sensitive manner, except that drebrin protein levels did not decrease after 3 days in DM; rather, they were still at their peak at this time point (Figures $1 \mathrm{~B}$ and $1 \mathrm{C}$ ). These results suggest that drebrin protein may be more stable than the mRNA that encodes it. In some Western blots, two distinct drebrin bands were resolved (e.g., Figure 1C). Treatment of cell lysates with alkaline phosphatase led to loss of the more slowly migrating band but had no effect on the mobility of the lower band, indicating that the upper band, as reported previously [45], is a phosphorylated form of drebrin (data not shown). The ratios of the two bands did not change as drebrin protein levels rose during differentiation, suggesting that phosphorylation is not a differentiation-dependent or regulated event.
RNAi-mediated depletion of drebrin inhibits myoblast differentiation and myotube formation

To explore a role for drebrin in myogenesis, an RNAi approach was taken. Two independent $D b n 1$ sequences were placed into the pSuper.puro vector and were individually and stably expressed in $\mathrm{C} 2 \mathrm{C} 12$ cells. A vector harboring an irrelevant sequence was used as a control. Induction of drebrin was substantially dampened during differentiation of $\mathrm{C} 2 \mathrm{C} 12$ cells that expressed either siRNA sequence, relative to control transfectants (Figure $2 \mathrm{C}$ ). Depletion of drebrin with either sequence resulted in a similar strong reduction in the formation of multinucleated myotubes. Whereas control cells formed large myotubes, the majority of which had more than 20 nuclei, drebrin-depleted cells formed thin myotubes with fewer than five nuclei (Figures $2 \mathrm{~A}$ and $2 \mathrm{~B}$ ). Production of the differentiation markers myogenin and $\mathrm{MHC}$ was somewhat variably affected in these cell lines, but each accumulated lower amounts of these muscle-specific proteins than did control transfectants during 3 days in DM (Figure 2C). To explore this further, we directly transfected siRNAs corresponding to a third $D b n 1$ sequence into both $\mathrm{C} 2 \mathrm{C} 12$ cells and primary myoblasts. In both cell types, drebrin protein was barely detectable 

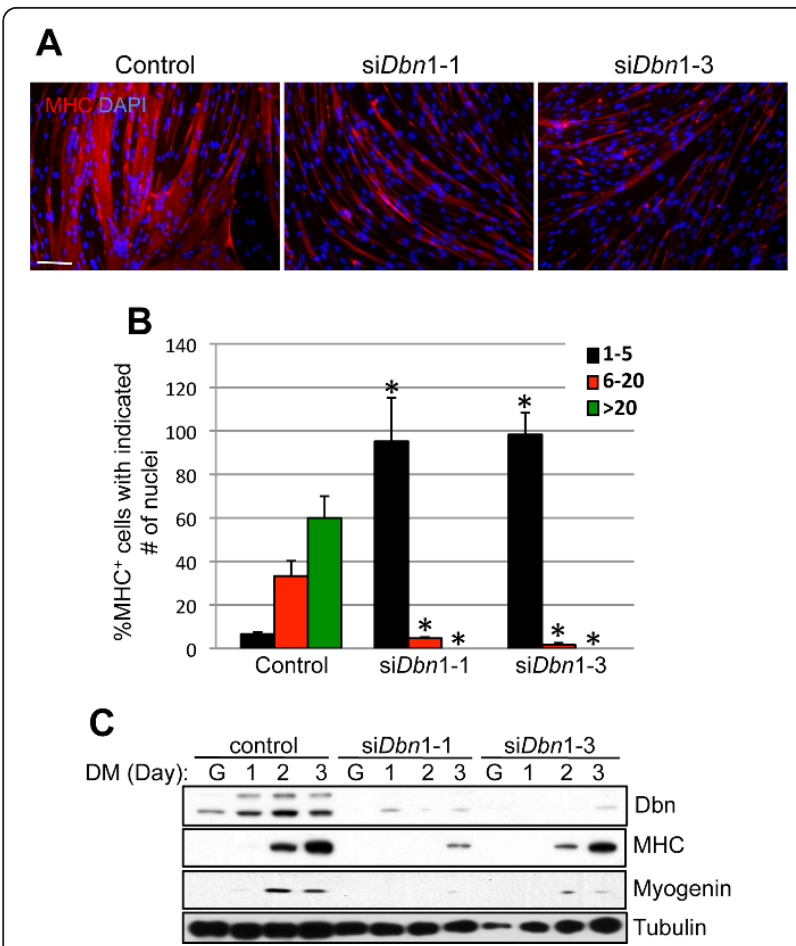

Figure 2 RNA interference (RNAi)-mediated depletion of drebrin reduces $\mathrm{C} 2 \mathrm{C} 12$ cell differentiation. (A) $\mathrm{C} 2 \mathrm{C} 12$ cells were transfected with either pSUPER (control) or pSUPER containing RNAi sequences targeted against Dbn 1 (siDbn 1-1, siDbn 1-3). After 3 days in differentiation medium (DM), cultures were fixed and doublestained for myosin heavy chain (red) and 4,6-diamidino-2-

phenylindole (blue). Bar: $0.1 \mathrm{~mm}$. (B) Quantification of myotube formation. Values shown are means \pm SD of triplicate determinations. ${ }^{*} P<0.05$. (C) Western blot analysis of $\mathrm{C} 2 \mathrm{C} 12$ cells expressing the indicated control or Dbn1 siRNA vectors cultured in growth medium (G) or DM for the indicated times.

after knockdown (Figures 3A, C, D and 3F). Expression of myogenin and MHC in these cultures was observed by Western blotting and immunofluorescence, but was diminished relative to control cultures (Figures 3A, C, D and $3 \mathrm{~F}$ ). Formation of multinucleated myotubes was strongly inhibited in both $\mathrm{C} 2 \mathrm{C} 12$ and primary myoblasts by anti-Dbn1 siRNA (Figures 3A, B, D and 3E). Taken together, these results indicate that drebrin promotes myoblast differentiation, as assessed by both biochemical and morphological criteria.

The possibility that defective differentiation of myoblasts depleted of drebrin was due to alterations in cell proliferation or apoptosis was examined by measuring BrdU incorporation and by terminal deoxynucleotidyltransferase deoxyuridine triphosphate nick-end labeling (TUNEL) assay, respectively. BrdU incorporation was slightly, but significantly, increased in primary myoblasts in GM treated with anti-Dbn1 siRNA relative to control cells (Figure 4A). Approximately $2 \%$ of cells in control cultures in GM were TUNEL-positive, and this was increased to about 5\% in drebrin-depleted cultures (Figure 4B). Forty-eight hours after switching to DM, about $8 \%$ of cells in the control cultures were TUNEL-positive, whereas approximately $15 \%$ of cells in drebrin-depleted cultures were undergoing apoptosis (Figure 4B). Although these increases in cell proliferation and apoptosis may contribute to the defective myogenesis seen in cells depleted of drebrin, they are insufficient to explain the quantitatively much stronger effects on myotube formation.

\section{The drebrin inhibitor BTP2 blocks myoblast differentiation and myotube formation}

BTP2 is a 3,5-bis(trifluoromethyl)pyrazole derivative that binds directly to drebrin and blocks actin rearrangements induced by forced expression of drebrin in $\mathrm{CHO}$ cells $[46,47]$. We treated $\mathrm{C} 2 \mathrm{C} 12$ cells and primary myoblasts with BTP2 to assess its effects on formation of myotubes and expression of myogenin and MHC. In both primary myoblasts and $\mathrm{C} 2 \mathrm{C} 12$ cells, treatment with BTP2 throughout a 3-day differentiation time course resulted in formation of myotubes with much fewer nuclei than those formed by vehicle-treated cells (Figures $5 \mathrm{~A}, \mathrm{~B}, \mathrm{D}$ and $5 \mathrm{E}$ ). Expression of myogenin and MHC was also diminished by BTP2 (Figures $5 \mathrm{C}$ and $5 F)$. Therefore, treatment of myoblasts with BTP2 produced a phenotype similar to knockdown of drebrin, although BTP2 did not alter drebrin protein levels (Figures $5 \mathrm{C}$ and $5 \mathrm{~F}$ ).

To prove that drebrin was the target of BTP2 that led to inhibition of myogenesis, $\mathrm{C} 2 \mathrm{C} 12$ cells were stably transfected with expression vectors encoding either wildtype drebrin $\mathrm{E}$ fused to GFP or a mutant form of drebrin E that cannot bind BTP2(K270M, K271M) [46], also fused to GFP. A vector that expressed GFP alone was used as an additional control. Western blot analyses with antibodies to drebrin showed that the levels of drebrin EGFP and drebrin E(K270M, K271M)-GFP were very similar to each other and to the levels of endogenously expressed drebrin (Figure 6A). As expected, GFP-expressing cells formed myotubes efficiently in a manner that was inhibited by treatment with BTP2 (Figures 6B and $6 C$ ). Expression of drebrin E-GFP had only a very modest ability to rescue the inhibitory effects of BTP2 (Figures $6 \mathrm{~B}$ and $6 \mathrm{C}$ ), suggesting that the concentration of the drug used was sufficient to inhibit the approximately twofold increase in overall drebrin levels in these cells. In contrast, expression of drebrin E(K270M, K271M)-GFP strongly rescued the fusion index of BTP2-treated cells to near that of untreated cells (Figures 6B and 6C). These results indicate that the major target of BTP2 in the inhibition of myogenesis is drebrin.

To gain further insight into drebrin function, 5-day (120 hours) differentiation time courses were performed 


\section{A}

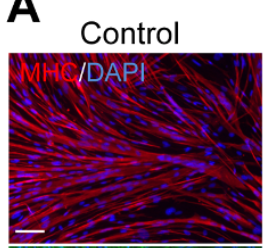

Dbn1 siRNA

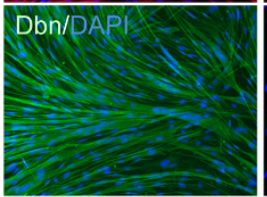

B

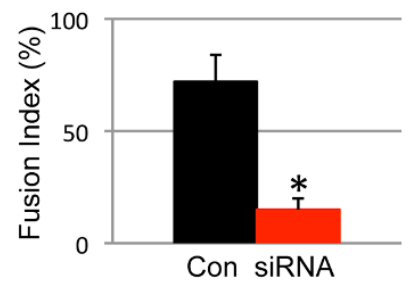

C

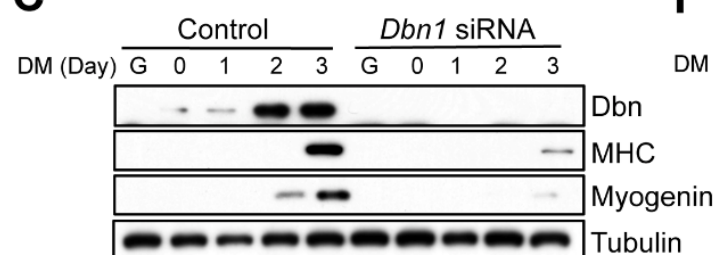

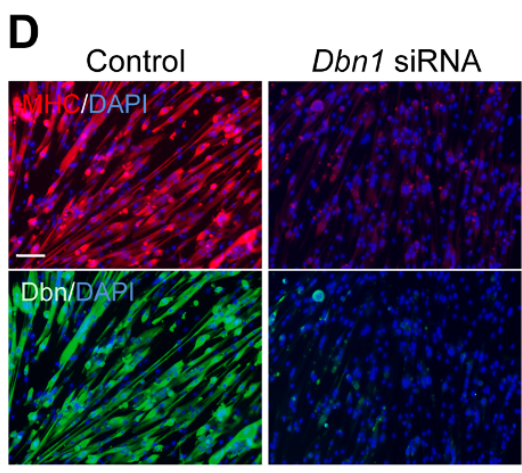

E

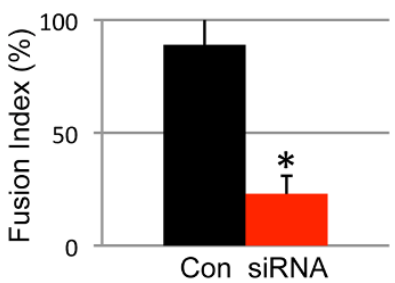

F

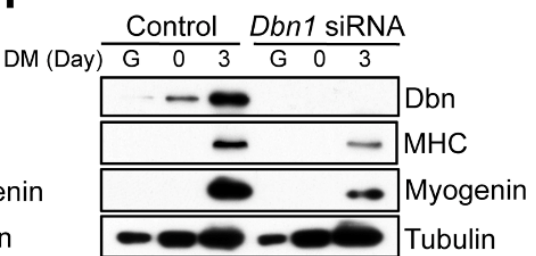

Figure 3 siRNA-mediated depletion of drebrin inhibits differentiation of primary myoblasts. (A) C2C12 cells were transfected with nonsilencing (control) or Dbn1-specific siRNA. After 3 days in differentiation medium, cultures were fixed and triple-stained for myosin heavy chain (MHC; red), drebrin (green) and 4',6-diamidino-2-phenylindole (blue). Bar: $20 \mu \mathrm{M}$. (B) Quantification of fusion index in cultures from part (A). Con, control siRNA. Values shown are means \pm SD of triplicate determinations. ${ }^{*} P<0.01$. (C) Cultures parallel to those in part (A) were examined by Western blot analysis for drebrin, myogenin and MHC production. Tubulin was used as a loading control. (D) through (F) are the same as parts (A) through (C), except primary myoblasts were used.

under the following conditions: (1) BTP2 was present in the cultures continuously from the time of switch to DM (designated as time 0), (2) BTP2 was added to cultures after 60 hours in DM without BTP2 and was maintained until the final 120-hour time point or (3) DMSO was present in the cultures as a vehicle control throughout the entire 120-hour time course (see Figure 7A). Cells were harvested at 72 and 120 hours for analysis of myotube formation and production of myogenin and MHC. Relative to DMSO vehicle controls, cells treated with BTP2 throughout the time course (condition 1) displayed a reduced overall fusion index, smaller myotubes with fewer nuclei and lower levels of myogenin and MHC at both 72 and 120 hours (Figures $7 \mathrm{~B}$ through $7 \mathrm{E}$ ). The number of nuclei per myotube, and levels of MHC, increased between 72 and 120 hours in DM in both control cultures and cultures continuously treated with BTP2, but the latter cultures differentiated defectively by these measures at both time points, relative to control cells at the same time point (Figures $7 \mathrm{~B}$ and $7 \mathrm{E})$. In contrast to the effects seen with cells treated continuously with BTP2, cultures in which BTP2 treatment began at 60 hours were indistinguishable from DMSO control cultures when analyzed at 120 hours. Levels of MHC (but not myogenin) increased in control cultures between 72 and 120 hours, but this increase was unaffected when BTP2 was added at the 60-hour time point (Figure 7B). Similarly, the number of nuclei per myotube increased significantly between 72 and 120 hours in control cultures, and myotubes with very large numbers of nuclei formed during this period; however, inclusion of BTP2 at 60 hours had no effect on this process (Figures $7 \mathrm{C}$ through $7 \mathrm{E}$ ). The overall fusion index of control cultures did not increase between 72 and 120 hours, so the increase in nuclei per myotube at 120 hours presumably reflects myotubemyotube fusion. The results shown in Figure 7 reveal that drebrin functions to promote myogenesis relatively 


\section{A}

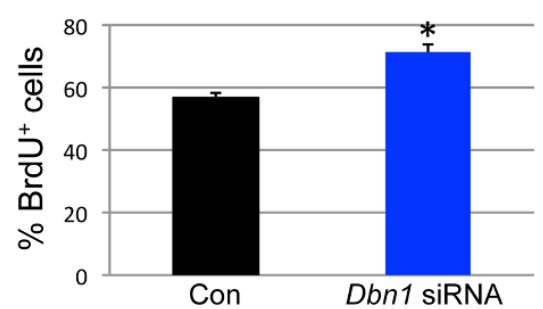

B

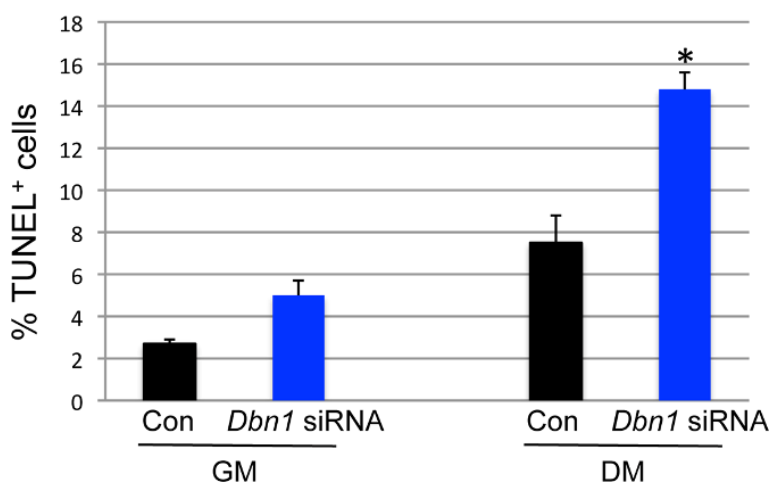

Figure 4 Cell proliferation and apoptosis in primary myoblasts depleted of drebrin. (A) Primary myoblatsts were transfected with control or Dbn1-specific siRNA. Cells were cultured for 36 hours in growth medium (GM), then exposed to bromodeoxyuridine (BrdU) for 2 hours and finally fixed and stained with antibody against BrdU. Values shown are means \pm SD of triplicate determinations. (B) Primary myoblasts were transfected with control (Con) or Dbn 1specific siRNA. Cells were cultured in GM for 36 hours or in differentiation medium for 48 hours. Values shown are means \pm SD of triplicate determinations. ${ }^{*} P<0.01$ by Student's $t$-test.

early in the differentiation process and that it is dispensable in a later phase characterized by increased levels of $\mathrm{MHC}$ and myotube-myotube fusion.

\section{Drebrin localization}

We determined drebrin's subcellular localization during differentiation of C2C12 myoblasts by immunofluorescence. Although drebrin levels are relatively low in lowdensity cultures in GM (Figure 1), drebrin was concentrated in cell projections and at cell cortices, areas that also accumulated F-actin, as revealed by costaining with phalloidin (Figure 8A). A similar result was obtained by direct visualization of drebrin E-GFP transiently expressed in $\mathrm{C} 2 \mathrm{C} 12$ cells (Figure $8 \mathrm{C}$ ). In higher-density cultures in DM for 24 hours (a condition in which such cells are preparing to fuse), drebrin was somewhat diffusely distributed in the cytoplasm but was concentrated at sites of cell-cell contact (Figure 8A). In myotubes after 3 days in DM, drebrin was again concentrated at sites of cell contact between myotubes and also at the tips of myotubes (Figure 8B).

\section{Discussion}

Myoblast differentiation involves expression of the muscle-specific transcriptional program and changes in cellular morphology, most strikingly among the latter, fusion into multinucleated myofibers. The p38 MAPK signaling pathway promotes myogenic differentiation, including myoblast fusion $[6,8]$. The reported substrates of p38 that are involved in myogenesis are all involved with expression of muscle-specific genes [11-14,16-18], yet few p38 target genes that regulate myogenesis have been identified. In this report, we identify $D b n 1$, encoding drebrin, as a gene that is induced during myoblast differentiation in an SB203580-sensitive manner and that promotes the differentiation process.

The inhibition of $D b n 1$ induction by SB203580 suggests that one or more p38-regulated transcription factors are involved, directly or indirectly, in the expression of $D b n 1$ during myoblast differentiation. MyoD and Mef2 family members are well-established as p38-regulated transcription factors that are central to musclespecific gene expression and myogenesis, and they are therefore potential candidate regulators of $D b n 1$ expression [8]. Investigators in a recent study identified MEK5/ERK5 as a signaling pathway in myoblasts that induces expression of the transcription factors Klf2 and Klf4. Klf2 and Klf4 in turn regulate a set of target genes involved in myoblast fusion, largely independently of MyoD and Mef2, and Dbn1 was among this set of ERK5 $\rightarrow$ Klf2/Klf4 target genes [48]. Klf4 can be posttranslationally activated by p38 MAPK [49], providing another potential explanation for the sensitivity of $\mathrm{Dbn} 1$ induction to SB203580. The nonreceptor tyrosine kinase FAK is important for myoblast maturation and fusion, and $D b n 1$ induction failed to occur properly in primary myoblasts in which fusion was inhibited by forced expression of FAT (Focal Adhesion Targeting, a naturally occurring dominant-negative variant of FAK) (see Table S1 in [9]). Taken together, $D b n 1$ expression has been associated with myogenesis in several systems, but its regulation is likely to be complex, integrating input from multiple signaling pathways and, potentially, multiple transcription factors.

RNAi-mediated depletion of drebrin or inhibition of drebrin function with the chemical inhibitor BTP2 led to decreases in expression of myogenin and $\mathrm{MHC}$ and inhibition of myotube formation. Drebrin encodes an Factin side-binding protein and is best known for its role in remodeling actin to promote maturation of filopodia into dendritic spines during synaptogenesis in developing neurons $[27,28]$. Production and remodeling of Factin-enriched structures is a key event in myoblast fusion [50], and it is logical to posit that drebrin may participate in such events. However, the fact that 


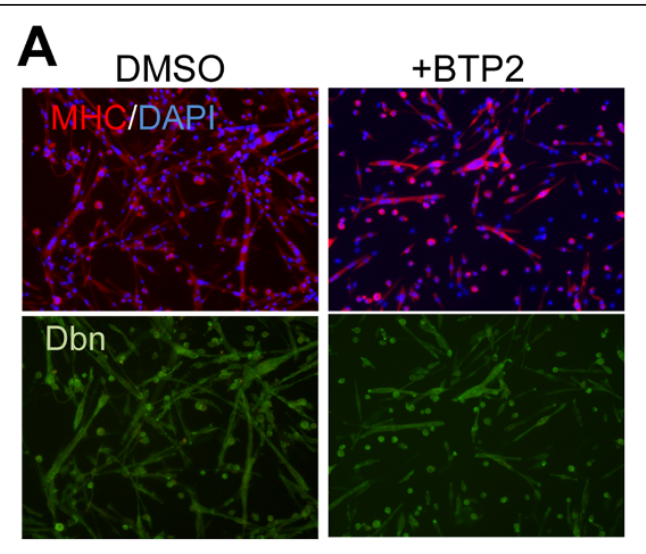

B

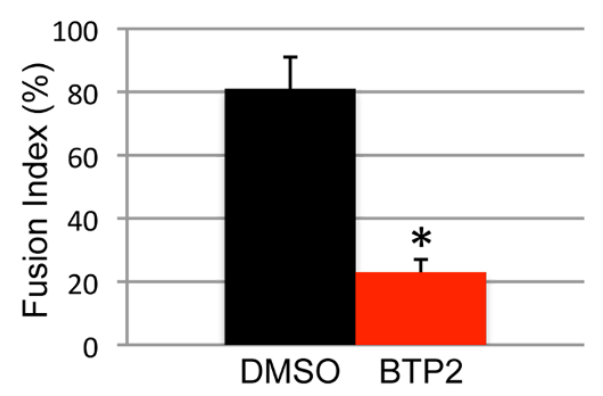

C

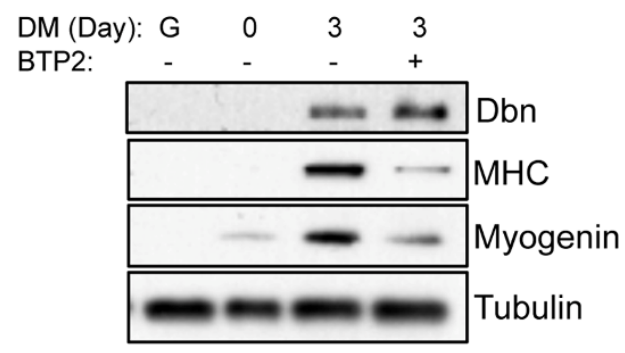

F

DM (Day): $\mathrm{G} \quad \begin{array}{lllllll}0 & 1 & 1 & 2 & 2 & 3 & 3\end{array}$

BTP2: $\quad-\quad-\quad+\quad+\quad+\quad-\quad+$

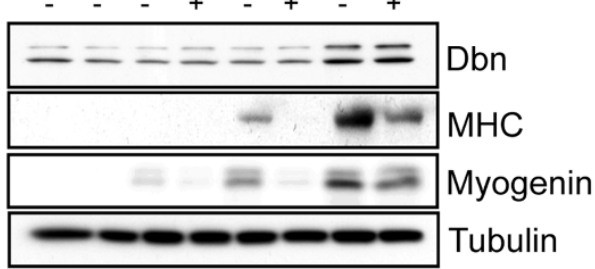

D
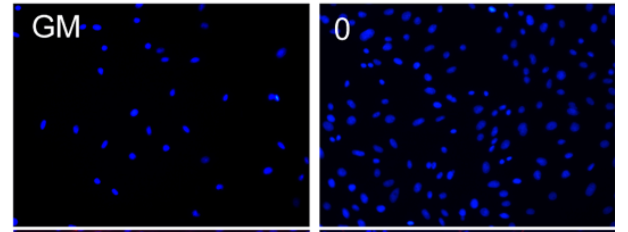

D1 DMSO

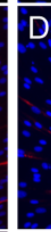
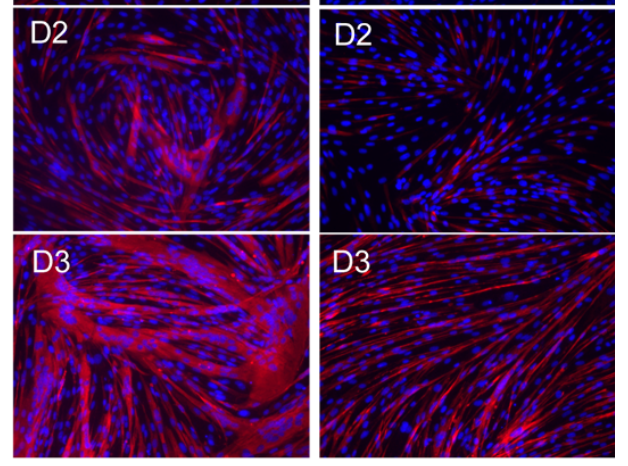

E
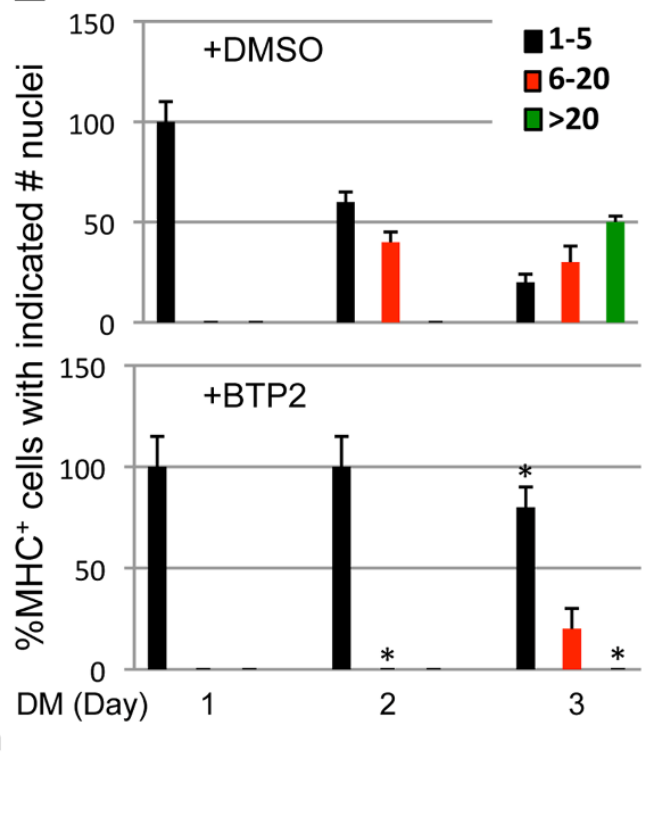

Figure 5 The drebrin antagonist BTP2 inhibits myoblast differentiation. (A) Primary myoblasts were cultured in differentiation medium for 72 hours in the presence or absence of the drebrin inhibitor BTP2 $(5 \mu \mathrm{M})$, then fixed and stained for myosin heavy chain (MHC) (red) and drebrin (green). Nuclei were counterstained with 4',6-diamidino-2-phenylindole (blue). (B) Quantification of the fusion index in cultures from part (A). Values shown are means \pm SD of triplicate determinations. ${ }^{*} P<0.01$. (C) Cultures parallel to those in part (A) were examined by Western blot analysis for drebrin, myogenin and MHC production. Tubulin was used as a loading control. (D) through (F) are the same as (A) through (C), except C2C12 cells were used. The asterisks in (E) refer to differences between dimethyl sulfoxide- and BTP2-treated cells in each of the indicated categories. 


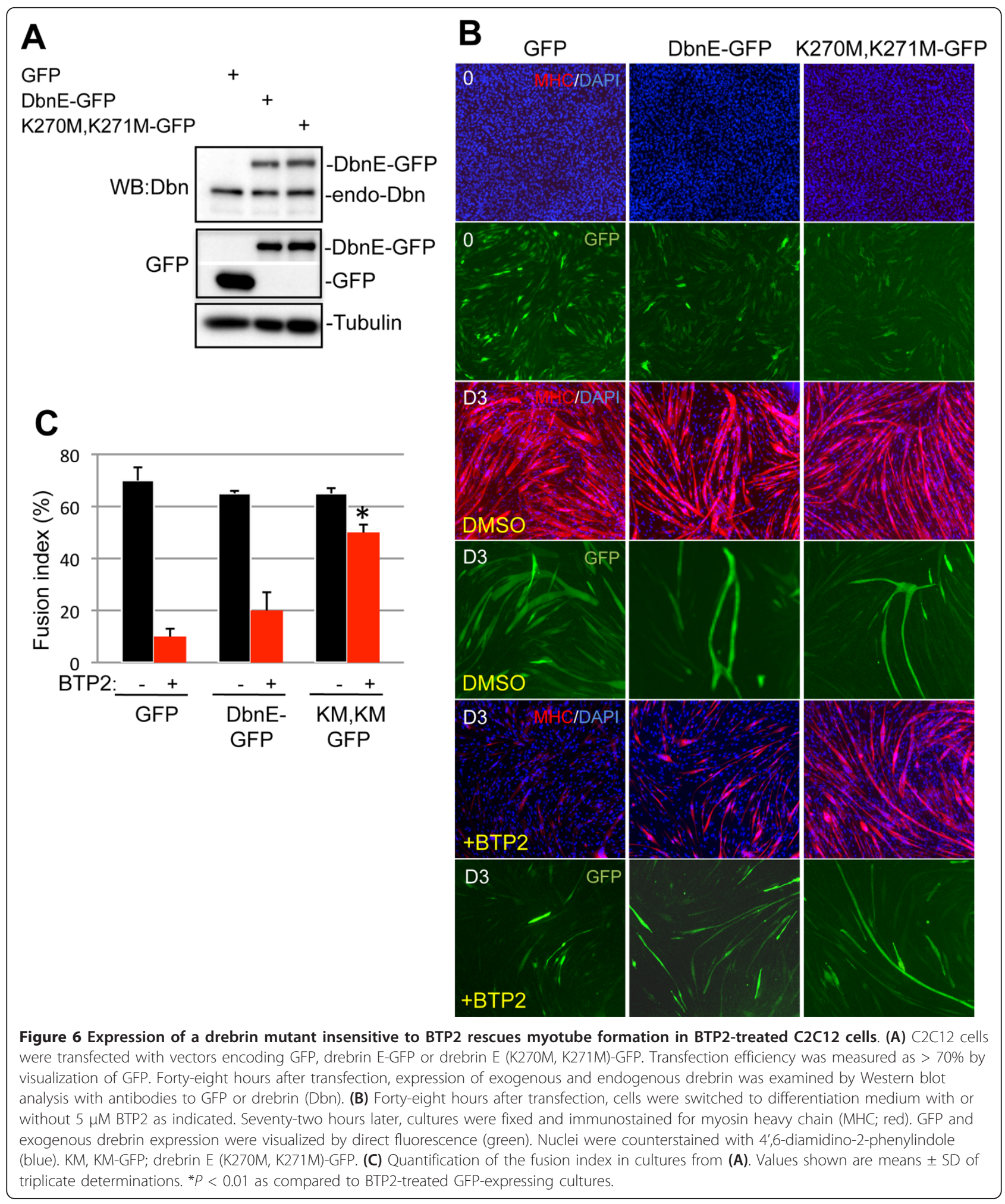

depletion or chemical inhibition of drebrin reduced the levels of myogenin, which is itself required for differentiation, raises the possibility that the diminished fusion into myotubes seen upon loss of drebrin function may occur as a consequence of a more generalized block in differentiation due to suboptimal myogenin induction. The decrease in MHC expression seen in drebrin-inhibited cells and the fact that, based on time courses of 


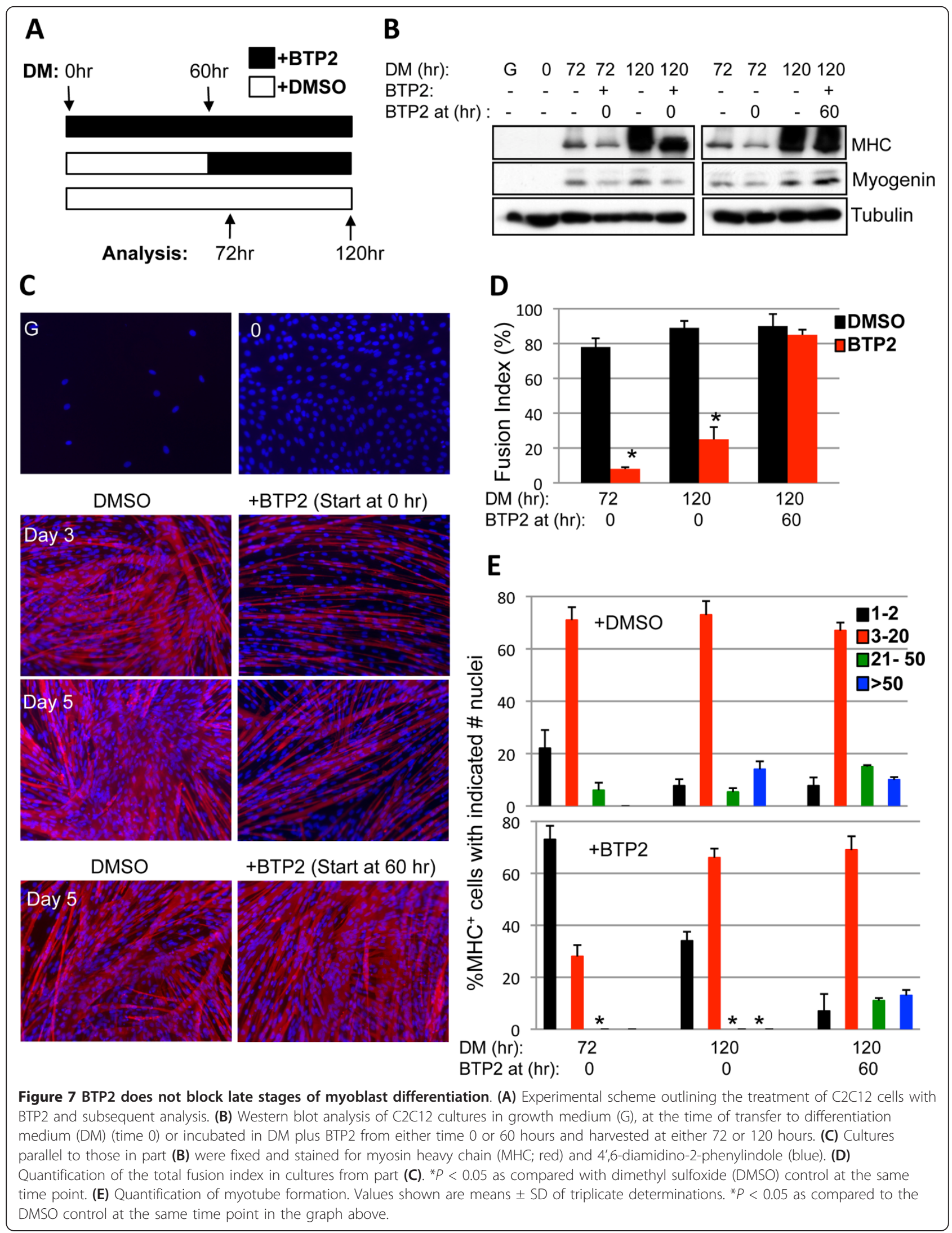




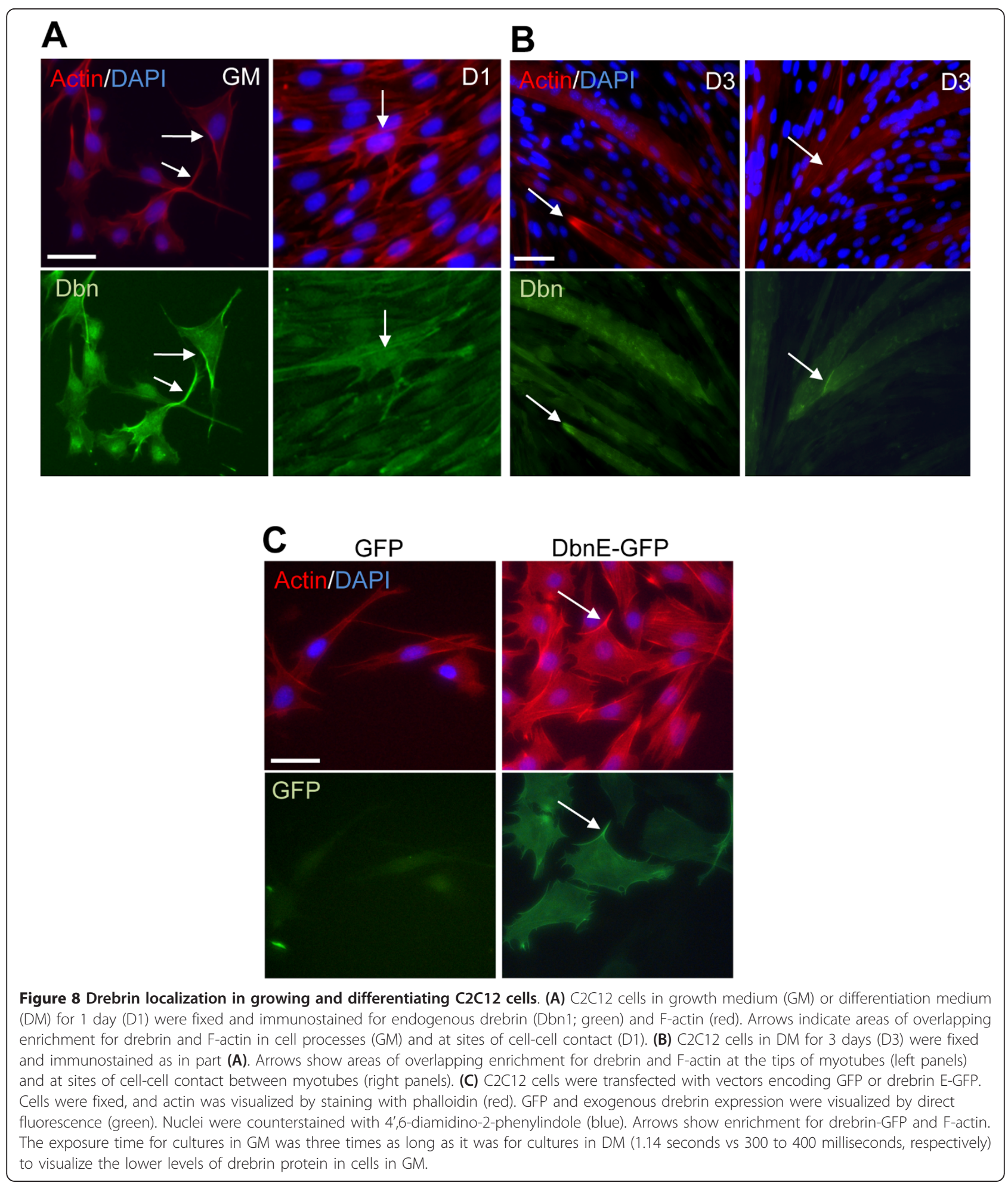

BTP2 treatment, drebrin function was not required after 60 hours in DM, supports the idea that regulation of myogenin expression is likely involved with at least some portion of drebrin's actions in myogenesis. Consistent with this notion, there is evidence to suggest that proper regulation of cytoskeletal structures may be important in muscle-specific gene expression. For example, muscle-specific gene expression is hindered when myoblasts are treated with the F-actin remodeling inhibitor latrunculin B or depleted of the microtubule- 
binding protein EB1 [21,24]. Additionally, Gussoni and colleagues [51] reported that C6ORF32, a cytoplasmic protein induced during myoblast differentiation that promotes filopodia formation, is important for expression of myogenin and MHC. Furthermore, depletion in cultured mammalian myoblasts of factors specific for myoblast fusion in Drosophila (for example, Dock1, Brag2) not only blocks formation of multinucleated cells but also decreases the percentage of cells that express muscle-specific markers [21,52]. It seems logical that at least some aspects of muscle-specific transcription and myoblast fusion would be coordinately regulated via cytoskeletal dynamics, and, if so, drebrin would be wellplaced to participate in such coordinated regulation. Consistent with this likelihood, our findings indicate that drebrin in myoblasts is enriched in cellular projections, cell cortices and regions of cell-cell contact, all sites where we found F-actin was also concentrated.

Drebrin interacts with several other proteins that have been implicated in myoblast differentiation and fusion. For example, drebrin binds the microtubule plus-tip binding protein EB3 in axonal growth cones. This interaction is important for growth cone formation and axonal growth and provides a link between F-actin and dynamic microtubules [30,33]. EB3 is important for myoblast fusion, and its expression is induced during myoblast differentiation with kinetics similar to $D b n 1$ induction [34]. Drebrin also binds to the scaffold protein Homer [35]. Homer2b promotes signaling by the calcineurin/Nuclear Factor of Activated T Cells (NFAT) pathway and enhances myoblast differentiation and myotube formation [36]. NFAT family members regulate several aspects of myogenesis [7]. Interestingly, the drebrin inhibitor BTP2 was originally identified in a screen for small-molecule antagonists of NFAT activity and was subsequently shown to bind directly to drebrin [47]. In addition to their ability to regulate calcium signaling, Homer proteins associate with Cdc42 [35], a small GTPase involved in myoblast differentiation and fusion $[37,38]$. Finally, drebrin binds to the cytoplasmic tail of the chemokine receptor CXCR4 [40]. CXCR4 promotes myoblast and myocyte migration [39]. Given the plethora of drebrin interactions of potential relevance, extensive structure-function studies are likely necessary to identify the most important ones for drebrin's actions in myogenesis.

\section{Conclusions}

In this study, we identified $D b n 1$ as a gene induced during myoblast differentiation in a p38 MAPK-dependent manner. $D b n 1$ encodes drebrin, an actin-binding protein that localizes to cellular extensions, cell cortices and sites of cell-cell contact. Inhibition of drebrin function, either by RNAi-mediated depletion or by the small- molecule inhibitor BTP2, reveals a role for drebrin in myogenic differentiation.

\section{Abbreviations}

DM: differentiation medium; DMEM: Dulbecco's modified Eagle's medium; GFP: green fluorescent protein; GM: growth medium; MHC: myosin heavy chain; PBS: phosphate-buffered saline; RNAi: RNA interference; RT-PCR: reverse transcriptase polymerase chain reaction; siRNA: small interfering RNA.

\section{Acknowledgements}

We are grateful to Min Lu and Mingi Hong for critiquing the manuscript. This work was supported by a grant from the National Institute of Arthritis and Musculoskeletal and Skin Diseases of the National Institutes of Health (AR46207; to RSK) and Grant-in-Aid for Scientific Research (A) from MEXT of Japan (19200029; to TS).

\section{Author details}

${ }^{1}$ Department of Developmental and Regenerative Biology, Mount Sinai School of Medicine, One Gustave L. Levy Place, New York, NY 10029, USA. ${ }^{2}$ Department of Medicine, Mount Sinai School of Medicine, One Gustave L. Levy Place, New York, NY 10029, USA. ${ }^{3}$ Department of Neurobiology and Behavior, Gunma University Graduate School of Medicine, 3-39-22, Showamachi, Maebashi, Gunma 371-8511, Japan.

\section{Authors' contributions}

AM, DS and RSK designed the experiments. AM and DS carried out the experiments. AM, DS, WZ and RSK analyzed the data. HY and TS generated and provided critical reagents. AM and RSK wrote the paper. All authors read and approved the final manuscript.

\section{Competing interests}

The authors declare that they have no competing interests.

Received: 19 August 2011 Accepted: 8 December 2011

Published: 8 December 2011

\section{References}

1. Biressi S, Molinaro $M$, Cossu G: Cellular heterogeneity during vertebrate skeletal muscle development. Dev Biol 2007, 308:281-293.

2. Charge SB, Rudnicki MA: Cellular and molecular regulation of muscle regeneration. Physiol Rev 2004, 84:209-238.

3. Pownall ME, Gustafsson MK, Emerson CP Jr: Myogenic regulatory factors and the specification of muscle progenitors in vertebrate embryos. Annu Rev Cell Dev Biol 2002, 18:747-783.

4. Tapscott SJ: The circuitry of a master switch: Myod and the regulation of skeletal muscle gene transcription. Development 2005, 132:2685-2695.

5. Tajbakhsh S, Buckingham M: The birth of muscle progenitor cells in the mouse: spatiotemporal considerations. Curr Top Dev Biol 2000, 48:225-268.

6. Guasconi $\mathrm{V}$, Puri PL: Chromatin: the interface between extrinsic cues and the epigenetic regulation of muscle regeneration. Trends Cell Biol 2009, 19:286-294.

7. Horsley V, Pavlath GK: NFAT: ubiquitous regulator of cell differentiation and adaptation. J Cell Biol 2002, 156:771-774.

8. Lluís F, Perdiguero E, Nebreda AR, Muñoz-Cánoves P: Regulation of skeletal muscle gene expression by p38 MAP kinases. Trends Cell Biol 2006, 16:36-44.

9. Quach NL, Biressi S, Reichardt LF, Keller C, Rando TA: Focal adhesion kinase signaling regulates the expression of caveolin 3 and $\beta 1$ integrin, genes essential for normal myoblast fusion. Mol Biol Cell 2009, 20:3422-3435.

10. Bergstrom DA, Penn BH, Strand A, Perry RL, Rudnicki MA, Tapscott SJ: Promoter-specific regulation of MyoD binding and signal transduction cooperate to pattern gene expression. Mol Cell 2002, 9:587-600.

11. de Angelis L, Zhao J, Andreucci JJ, Olson EN, Cossu G, McDermott JC: Regulation of vertebrate myotome development by the p38 MAP kinase-MEF2 signaling pathway. Dev Biol 2005, 283:171-179.

12. Perdiguero E, Ruiz-Bonilla V, Gresh $L$, Hui L, Ballestar E, Sousa-Victor $P$, Baeza-Raja B, Jardí M, Bosch-Comas A, Esteller M, Caelles C, Serrano AL, Wagner EF, Muñoz-Cánoves P: Genetic analysis of p38 MAP kinases in 
myogenesis: fundamental role of p38a in abrogating myoblast proliferation. EMBO J 2007, 26:1245-1256.

13. Wu Z, Woodring PJ, Bhakta KS, Tamura K, Wen F, Feramisco JR, Karin M, Wang JY, Puri PL: p38 and extracellular signal-regulated kinases regulate the myogenic program at multiple steps. Mol Cell Biol 2000, 20:3951-3964.

14. Briata P, Forcales SV, Ponassi M, Corte G, Chen CY, Karin M, Puri PL, Gherzi R: p38-dependent phosphorylation of the mRNA decaypromoting factor KSRP controls the stability of select myogenic transcripts. Mol Cell 2005, 20:891-903.

15. Lluís F, Ballestar E, Suelves M, Esteller M, Muñoz-Cánoves P: E47 phosphorylation by p38 MAPK promotes MyoD/E47 association and muscle-specific gene transcription. EMBO J 2005, 24:974-984

16. Rampalli S, Li L, Mak E, Ge K, Brand M, Tapscott SJ, Dilworth FJ: p38 MAPK signaling regulates recruitment of Ash2L-containing methyltransferase complexes to specific genes during differentiation. Nat Struct Mol Biol 2007, 14:1150-1156.

17. Serra C, Palacios D, Mozzetta C, Forcales SV, Morantte I, Ripani M, Jones DR, Du K, Jhala US, Simone C, Puri PL: Functional interdependence at the chromatin level between the MKK6/p38 and IGF1/PI3K/AKT pathways during muscle differentiation. Mol Cell 2007, 28:200-213.

18. Simone C, Forcales SV, Hill DA, Imbalzano AN, Latella L, Puri PL: p38 pathway targets SWI-SNF chromatin-remodeling complex to musclespecific loci. Nat Genet 2004, 36:738-743.

19. Abramovici $\mathrm{H}$, Gee SH: Morphological changes and spatial regulation of diacylglycerol kinase- $\zeta$, syntrophins, and Rac1 during myoblast fusion. Cell Motil Cytoskeleton 2007, 64:549-567.

20. Kim SJ, Kim S, Shin H, Uhm CS: Intercellular interaction observed by atomic force microscopy. Ultramicroscopy 2008, 108:1148-1151.

21. Nowak SJ, Nahirney PC, Hadjantonakis AK, Baylies MK: Nap1-mediated actin remodeling is essential for mammalian myoblast fusion. $J$ Cell Sci 2009, 122:3282-3293

22. Chang W, Webster DR, Salam AA, Gruber D, Prasad A, Eiserich JP, Bulinski JC: Alteration of the C-terminal amino acid of tubulin specifically inhibits myogenic differentiation. J Biol Chem 2002, 277:30690-30698.

23. O'Connor RS, Steeds CM, Wiseman RW, Pavlath GK: Phosphocreatine as an energy source for actin cytoskeletal rearrangements during myoblast fusion. J Physiol 2008, 586:2841-2853.

24. Zhang T, Zaal KJ, Sheridan J, Mehta A, Gundersen GG, Ralston E: Microtubule plus-end binding protein EB1 is necessary for muscle cell differentiation, elongation and fusion. J Cell Sci 2009, 122:1401-1409.

25. Ishikawa R, Hayashi K, Shirao T, Xue Y, Takagi T, Sasaki Y, Kohama K: Drebrin, a development-associated brain protein from rat embryo, causes the dissociation of tropomyosin from actin filaments. J Biol Chem 1994, 269:29928-29933.

26. Shirao T, Kojima N, Obata K: Cloning of drebrin $A$ and induction of neurite-like processes in drebrin-transfected cells. Neuroreport 1992, 3:109-112.

27. Majoul I, Shirao T, Sekino Y, Duden R: Many faces of drebrin: from building dendritic spines and stabilizing gap junctions to shaping neurite-like cell processes. Histochem Cell Biol 2007, 127:355-361.

28. Sekino Y, Kojima N, Shirao T: Role of actin cytoskeleton in dendritic spine morphogenesis. Neurochem Int 2007, 51:92-104.

29. Ikeda K, Kaub PA, Asada H, Uyemura K, Toya S, Shirao T: Stabilization of adhesion plaques by the expression of drebrin A in fibroblasts. Brain Res Dev Brain Res 1996, 91:227-236.

30. Mizui T, Kojima N, Yamazaki H, Katayama M, Hanamura K, Shirao T: Drebrin $E$ is involved in the regulation of axonal growth through actin-myosin interactions. J Neurochem 2009, 109:611-622.

31. Peitsch WK, Grund C, Kuhn C, Schnölzer M, Spring H, Schmelz M, Franke WW: Drebrin is a widespread actin-associating protein enriched at junctional plaques, defining a specific microfilament anchorage system in polar epithelial cells. Eur J Cell Biol 1999, 78:767-778,

32. Peitsch WK, Hofmann I, Prätzel S, Grund C, Kuhn C, Moll I, Langbein L, Franke WW: Drebrin particles: components in the ensemble of proteins regulating actin dynamics of lamellipodia and filopodia. Eur J Cell Biol 2001, 80:567-579.

33. Geraldo S, Khanzada UK, Parsons M, Chilton JK, Gordon-Weeks PR: Targeting of the F-actin-binding protein drebrin by the microtubule plus-tip protein EB3 is required for neuritogenesis. Nat Cell Biol 2008, 10:1181-1189
34. Straube A, Merdes A: EB3 regulates microtubule dynamics at the cell cortex and is required for myoblast elongation and fusion. Curr Biol 2007, 17:1318-1325.

35. Shiraishi-Yamaguchi Y, Sato Y, Sakai R, Mizutani A, Knöpfel T, Mori N, Mikoshiba K, Furuichi T: Interaction of Cupidin/Homer2 with two actin cytoskeletal regulators, Cdc42 small GTPase and Drebrin, in dendritic spines. BMC Neurosci 2009, 10:25

36. Stiber JA, Tabatabaei N, Hawkins AF, Hawke T, Worley PF, Williams RS, Rosenberg P: Homer modulates NFAT-dependent signaling during muscle differentiation. Dev Biol 2005, 287:213-224.

37. Vasyutina E, Martarelli B, Brakebusch C, Wende H, Birchmeier C: The small G-proteins Rac1 and Cdc42 are essential for myoblast fusion in the mouse. Proc Natl Acad Sci USA 2009, 106:8935-8940.

38. Kang JS, Bae GU, Yi MJ, Yang YJ, Oh JE, Takaesu G, Zhou YT, Low BC, Krauss RS: A Cdo/Bnip-2/Cdc42 signaling pathway regulates $p 38 \alpha / \beta$ MAPK activity and myogenic differentiation. J Cell Biol 2008, 182:497-507.

39. Griffin CA, Apponi LH, Long KK, Pavlath GK: Chemokine expression and control of muscle cell migration during myogenesis. J Cell Sci 2010, 123:3052-3060

40. Pérez-Martínez M, Gordón-Alonso M, Cabrero JR, Barrero-Villar M, Rey M, Mittelbrunn M, Lamana A, Morlino G, Calabia C, Yamazaki H, Shirao T, Vázquez J, González-Amaro R, Veiga E, Sánchez-Madrid F: F-actin-binding protein drebrin regulates CXCR4 recruitment to the immune synapse. $J$ Cell Sci 2010, 123:1160-1170.

41. Rando TA, Blau HM: Primary mouse myoblast purification, characterization, and transplantation for cell-mediated therapy. J Cell Biol 1994, 125:1275-1287.

42. Cole F, Zhang W, Geyra A, Kang JS, Krauss RS: Positive regulation of myogenic bHLH factors and skeletal muscle development by the cell surface receptor CDO. Dev Cell 2004, 7:843-854.

43. Kang JS, Mulieri PJ, Miller C, Sassoon DA, Krauss RS: CDO, a Robo-related cell surface protein that mediates myogenic differentiation. J Cell Biol 1998, 143:403-413.

44. Bae GU, Yang YJ, Jiang G, Hong M, Lee HJ, Tessier-Lavigne M, Kang JS, Krauss RS: Neogenin regulates skeletal myofiber size and focal adhesion kinase and extracellular signal-regulated kinase activities in vivo and in vitro. Mol Biol Cell 2009, 20:4920-4931

45. Chew CS, Okamoto CT, Chen X, Thomas R: Drebrin E2 is differentially expressed and phosphorylated in parietal cells in the gastric mucosa. Am J Physiol Gastrointest Liver Physiol 2005, 289:G320-G331.

46. Mercer JC, Qi Q, Mottram LF, Law M, Bruce D, lyer A, Morales JL, Yamazaki H, Shirao T, Peterson BR, August A: Chemico-genetic identification of drebrin as a regulator of calcium responses. Int Biochem Cell Biol 2010, 42:337-345.

47. Trevillyan J, Chiou XG, Chen YW, Ballaron SJ, Sheets MP, Smith ML, Wiedeman PE, Warrior U, Wilkins J, Gubbins EJ, Gagne GD, Fagerland J, Carter GW, Luly JR, Mollison KW, Djuric SW: Potent inhibition of NFAT activation and T cell cytokine production by novel low molecular weight pyrazole compounds. J Biol Chem 2001, 276:48118-48126.

48. Sunadome K, Yamamoto T, Ebisuya M, Kondoh K, Sehara-Fujisawa A Nishida E: ERK5 regulates muscle cell fusion through Klf transcription factors. Dev Cell 2011, 15:192-205.

49. Li HX, Han M, Bernier M, Zheng B, Sun SG, Su M, Zhang R, Fu JR, Wen JK: Krüppel-like factor 4 promotes differentiation by transforming growth factor- $\beta$ receptor-mediated Smad and p38 MAPK signaling in vascular smooth muscle cells. J Biol Chem 2010, 285:17846-17856.

50. Richardson BE, Nowak SJ, Baylies MK: Myoblast fusion in fly and vertebrates: new genes, new processes and new perspectives. Traffic 2008, 9:1050-1059.

51. Yoon S, Molloy MJ, Wu MP, Cowan DB, Gussoni E: C6ORF32 is upregulated during muscle cell differentiation and induces the formation of cellular filopodia. Dev Biol 2007, 301:70-81.

52. Pajcini KV, Pomerantz JH, Alkan O, Doyonnas R, Blau HM: Myoblasts and macrophages share molecular components that contribute to cell-cell fusion. J Cell Biol 2008, 180:1005-1019.

doi:10.1186/2044-5040-1-36

Cite this article as: Mancini et al: Regulation of myotube formation by the actin-binding factor drebrin. Skeletal Muscle 2011 1:36. 